\title{
Trimodal electroencephalogram during isoflurane anaesthesia
}

\author{
Lorenzo Dimpel
}

\begin{abstract}
The effects of common anaesthetic agents, such as isoflurane or propofol, on the electroencephalogram are well-known. The most characteristic feature is the marked increase in delta power and alpha power resulting in a bimodal power spectrum from light to moderately deep anaesthesia. Here, a case is presented where the power spectrum became trimodal when anaesthesia was lightened towards wake up. The implications of a third mode for depth-of-anaesthesia monitoring are discussed. Its neuro-physiology is unknown, as it has never been reported before.
\end{abstract}

Key words: Anaesthesia, bimodal, electroencephalogram, trimodal

\section{INTRODUCTION}

Effects of anaesthetic drugs on the electroencephalogram (EEG) were first described in 1931 and 1933 by Berger ${ }^{[1,2]}$ and by Gibbs et al. in 1937. ${ }^{[3]}$ Since then, innumerable reports have detailed the effects on the EEG of many anaesthetic drugs. Most of the currently used anaesthetics, such as propofol, isoflurane, thiopental and etomidate have similar EEG effects, despite their different molecular structures and diverse effects on a range of neuronal ion channels, and other subcellular structures. However, to some extent, they share one common mechanism, they are agonists on the Gamma-aminobutyric acid Type $A\left(G_{A B A}\right)$ receptor. For this reason, they are often referred to as the 'GABAergic' agents.

The EEG effects of these agents have been investigated and reviewed ${ }^{[4]}$ in detail:

Department of Anaesthesia, Derriford Hospital, Plymouth Hospitals NHS Trust, Plymouth, UK

Address for correspondence:

Dr. Lorenz Dimpel, Department of Anaesthesia,

Derriford Hospital, Level 9, Terrence Lewis Building,

Plymouth PL6 8DH, UK.

E-mail: lorenzodimpel@nhs.net

\begin{tabular}{|l|l|}
\hline \multicolumn{2}{|c|}{ Access this article online } \\
\hline Quick Response Code: & Website: \\
\hline & www.jnaccjournal.org \\
\cline { 2 - 2 } & \\
\hline & \\
\hline
\end{tabular}

From consciousness to deep anaesthesia, there is always prominent activity in the delta $(\Delta ; 0-4 \mathrm{~Hz})$ range, even though during wakefulness, this is not visible from the raw trace.

With subanaesthetic concentrations, so-called 'paradoxical activation' is seen in the frontal lobes. This activation consists of a significant increase in power across most of the alpha $(\alpha ; 8-13 \mathrm{~Hz})$ and beta $(\beta ; 13-30 \mathrm{~Hz})$ ranges, leading to a broad-based second peak (more graphically described as broad hump) in the power spectrum. Thus, the power distribution in the power spectrum has become bimodal (i.e., two peaks, in the $\Delta$ and the $\alpha / \beta$ range).

With increasing anaesthetic concentration, a progressive reduction in band width of the second peak, as well as a shift to lower frequencies is seen. At loss of consciousness, the $\beta$-power has almost returned to the awake level, whilst the second peak now lies mostly in the $\alpha$ range, with a maximal power around $12 \mathrm{~Hz} \cdot{ }^{[5]}$ The $\alpha$-power is increased approximately 20 -fold compared to the awake state. ${ }^{[6]}$

This is an open access article distributed under the terms of the Creative Commons Attribution-NonCommercial-ShareAlike 3.0 License, which allows others to remix, tweak, and build upon the work non-commercially, as long as the author is credited and the new creations are licensed under the identical terms.

For reprints contact: reprints@medknow.com

How to cite this article: Dimpel L. Trimodal electroencephalogram during isoflurane anaesthesia. J Neuroanaesthesiol Crit Care 2016;3:133-6. 
With further deepening of anaesthesia, prominent very low frequency $\Delta$ waves spread from the frontal lobe across the whole cortex, with a power approximately 20 times that of the awake state. ${ }^{[7]}$ At the same time, the $\alpha$ peak loses power and shifts down into the theta $(\Theta ; 4-8 \mathrm{~Hz})$ range, and may eventually disappear or melt into the $\Delta$ peak. Thus, the spectrum may eventually become mono-modal again. This well-established pattern has been labelled 'frequency progression pattern' (FPP). ${ }^{[7]}$

During clinical anaesthesia, anaesthesiologists usually aim at a rapid onset of deep anaesthesia by giving an intravenous bolus injection of anaesthetic agent. This will achieve high brain concentrations so rapidly, that the spectral changes cannot easily be appreciated. However, at discontinuation of anaesthesia, with gradually falling brain levels of anaesthetic agent, the above-described FPP can be observed in reverse order. ${ }^{[8]}$

These normal, well-known EEG features are illustrated in Figures 1 and 2, recorded during progressive lightening of isoflurane anaesthesia. In these screenshots both, the raw EEG trace and a spectral array are displayed. In the spectral array (top panel on the left), frequency is shown on the $\mathrm{X}$-axis and time (in minutes) on the $\mathrm{Y}$-axis, where zero denotes the current moment in time ('time zero') and 10 refers to the time $10 \mathrm{~min}$ ago (or ' $-10 \mathrm{~min}^{\prime}$ ). Power is shown in a colour scale. The spectral edge frequency (SEF; usually defined as the frequency below which $95 \%$ of the total EEG power lie) is shown as narrow black lines. In most of the spectral array shown in Figure 1, the second mode can barely be appreciated; however, during the last $2 \mathrm{~min}$ (bottom end of spectral

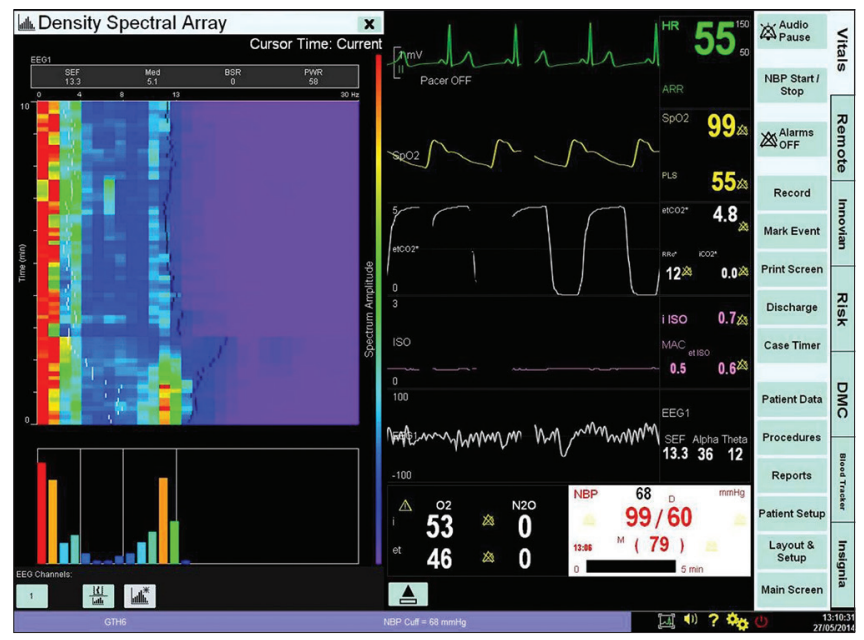

Figure 1: Screenshot from anaesthetic monitor. The right side shows the traces typically monitored during anaesthesia; the second but last is the raw electroencephalogram. Top left is the spectral array, with frequency shown on the X-axis, and time (the preceding $10 \mathrm{~min}$ being shown) on the Y-axis. Time 'zero' is the current moment in time. Power is expressed in a colour scale. Bottom left shows the most up-to-date power spectrum (X-axis frequency, Y-axis power) array), the second mode becomes much more prominent due to lightening of anaesthesia. In Figure 2 - taken 8 min after Figure 1 - the bimodal power distribution is very obvious. From Figure 1 to Figure 2, $\Delta$-power is fading, while the second peak is gaining power, with its peak frequency shifting upwards in the spectrum.

\section{CASE REPORT}

Here, I am presenting a case which clearly deviated from the well-established FPP as described above, demonstrating a trimodal EEG during lightening of isoflurane anaesthesia.

A 29-year-old, otherwise entirely healthy male patient was anaesthetised for a lumbar nerve root decompression, receiving fentanyl and propofol for induction of anaesthesia and isoflurane for maintenance. Anaesthesia and surgery were uneventful.

EEG was recorded throughout with the Draeger Infinity ${ }^{\circledR}$ EEG Pod and Infinity Delta monitor and displayed with the Draeger Infinity ${ }^{\circledR}$ Explorer software on a separate monitor (Draeger Medical UK Ltd., Hemel Hempstead, UK). Electrodes were placed at approximately AF3 - Fpz (international 10-10 system), with a mastoid electrode as ground. Depth of anaesthesia was monitored using the raw EEG and its power spectrum, and kept at a deep, monomodal level during the operation.

Near completion of surgery, the administration of isoflurane was discontinued, but end-expiratory concentration 'clamped' at a $0.4 \%$ by keeping the fresh gas flow to the circle system at a low level. Within minutes, the EEG became trimodal, with the second mode lying in the $\Theta$ range, whereas the third mode initially appeared in the $\alpha$ range, but within a few more minutes shifted into $\beta$ range, indicating a falling

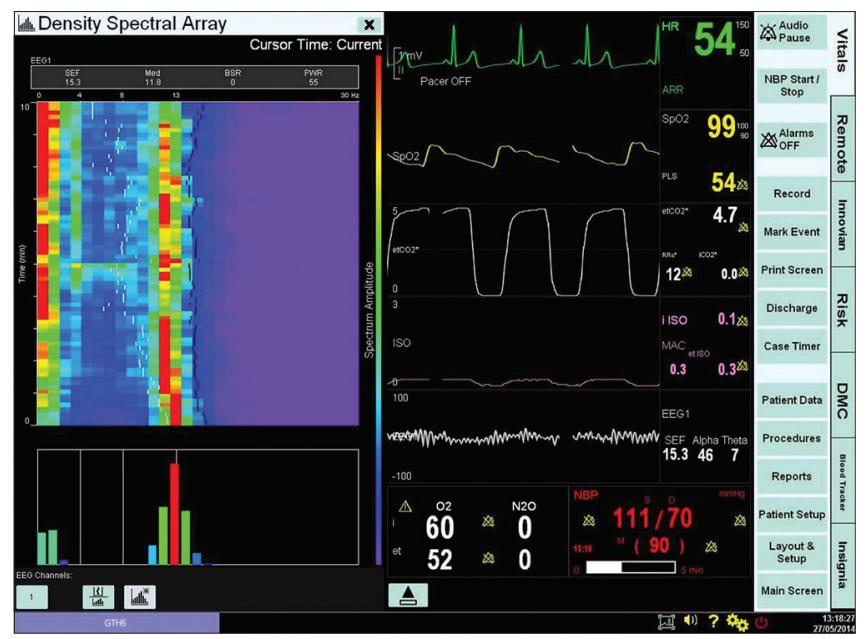

Figure 2: Screenshot from anaesthetic monitor. Taken $8 \mathrm{~min}$ after that of Figure 1. Monitor display settings identical to Figure 1 
effect site concentration (at steady-state end-tidal concentration) of isoflurane.

As this finding was unexpected, a memory device to download images directly from the anaesthetic monitor was not at hand, hence photographs with a cell phone camera were taken, which is responsible for the inferior image quality in Figures 3 and 4 . Of note, the bar graphs below the spectral array in these images refer to the time of the cursor (horizontal pale yellow line) in the spectral array, not the time zero. In Figure 3, the cursor lies at approximately $-3.25 \mathrm{~min}$, at a time just before the EEG became trimodal, thus, the bar graph does not demonstrate the trimodal power distribution, which is clearly visible in the last $3 \mathrm{~min}$ in the spectral array. In Figure 4, the cursor lies near time zero; consequently, the bar graph also depicts the trimodal spectrum.

\section{DISCUSSION}

As a micro volt signal, the EEG is highly susceptible to artefacts - therefore, artefact always needs to be considered as the cause of an unexpected EEG trace. In this instance, inspection of the raw trace does not suggest artefact as the source of the unusual FPP. Further, the power spectrum shows that the second and third modes have a broad frequency base. Persistent mechanical or electric artefacts in the operating room, on the other hand, tend to have a narrow frequency band, or even a single frequency, such as that of an electric or pneumatic drill. Perhaps, the strongest argument against an artefact is that the SEF is steadily rising from approximately $11 \mathrm{~Hz}$ to around $20 \mathrm{~Hz}$ over the $10 \mathrm{~min}$ shown in the density spectral array (little black lines; best appreciated in Figure 3), which is highly characteristic for anaesthetic

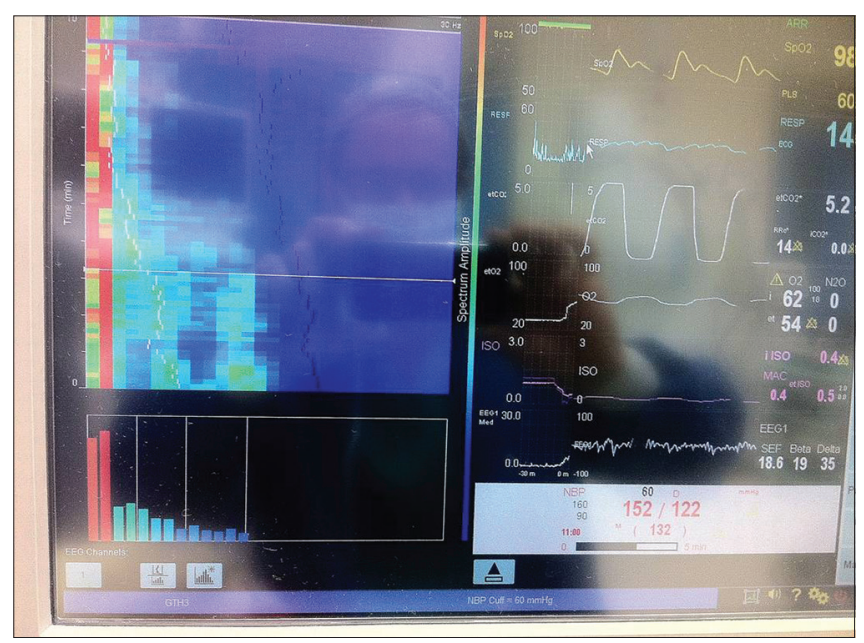

Figure 3: Photograph of anaesthetic monitor. Monitor display settings are as in Figure 1. The off-white line across the spectral array represents the trend curser, which is set at approximately 3.25 min before time zero - the bar graph power spectrum below relates to the time of the curser agent wash-out. An artefact would have thrown the SEF out of its course. In fact, a small and short-lasting artefact can be seen to deviate the SEF at about $-9 \mathrm{~min}$.

I am therefore confident that this trace represents true EEG. To my knowledge, this is the first report of a trimodal EEG in the context of light anaesthesia.

The neuro-physiological processes causing this trimodal power spectrum are unknown at this time.

The second mode in a typical, bimodal EEG consists of relatively high power and highly coherent $\alpha$ waves, believed to be generated by oscillation within the cortico-thalamic and thalamo-cortical pathways. ${ }^{[9]}$ In the case described here, it is the third mode that corresponds to the usual second mode in a typical EEG, according to its position in the power spectrum at the given level of anaesthesia. The second i.e. additional mode has a frequency half that of the third, as can be seen from Figure 4 . This might tempt to speculate, whether the second mode is the sub-harmonic of the third, arising from one and the same system. Sub-harmonics arising in the frontal lobe have been described, albeit in a completely different context i.e., in awake subjects during photic stimulation frequency locked to their own alpha rhythm. ${ }^{[10]}$ Given the proposed mechanism underlying the anaesthesia-induced $\alpha$ waves, ${ }^{[9]}$ it seems unlikely that this is the origin of the second mode here.

Alternatively, a separate 'pacemaker' or oscillating loop could be postulated.

Propofol and fentanyl, the other two centrally acting drugs which the patient had received, can be excluded as the cause of the unusual EEG: Opiates, as an adjunct to propofol or isoflurane anaesthesia, have limited effects on the FPP and are not known to cause trimodal patterns. ${ }^{[11]}$ Propofol causes an EEG pattern almost identical to that

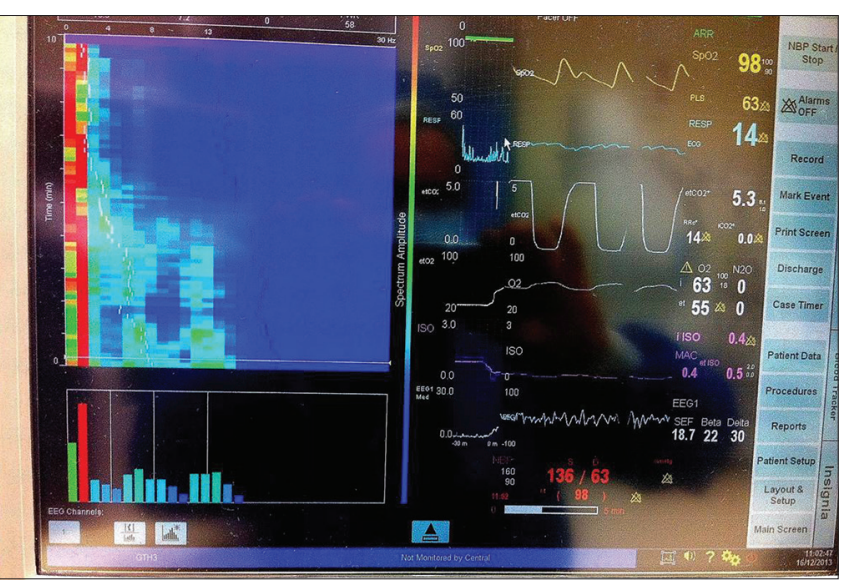

Figure 4: Photograph of anaesthetic monitor. Image taken approximately $30 \mathrm{~s}$ after the image in Figure 3; settings as in Figure 3, except for the curser which lies at approximately $20 \mathrm{~s}$ before time zero 
of isoflurane,,$^{[4]}$ and its brain level towards the end of the operation would have been negligible.

What are the clinical implications of this unusual EEG pattern? First of all, as the patient was entirely healthy and free from neurological disorders (other than compression of a lumbar nerve root), it should be recognised as a variant, rather than a sign of pathology, similar to well-known variants in the awake EEG. The anaesthetist monitoring raw EEG, the linear power spectrum, or both, must recognise it as a pattern indicating light anaesthesia.

The implications for 'depth of anaesthesia' monitoring with automated devices are potentially serious. These monitors are not based on underlying neuro-physiological mechanisms of consciousness or anaesthesia. The most widely used, EEG based monitor, the BIS ${ }^{\mathrm{TM}}$ (Bispectral Index monitor, Medtronic, Minneapolis, Minnesota), classifies the EEG in numbers between 0 and 100, where 100 indicates full consciousness and alertness, whereas 0 correlates to a flat line EEG. When last publicised, ${ }^{[4]}$ the classification algorithms were based on the ratios of power, and bispectral power, in empirically chosen frequency bands (and quantification of burst suppression when present). The here reported additional mode substantially increases power in a relatively low frequency band, included in the power ratios (when last publicised) used by the BIS, thus changing the value of those ratios. It is thus likely that the BIS would mis-classify the here reported trimodal EEG as relatively deep anaesthesia, even though it occurred at a relatively light state. However, the algorithms of the BIS have been modified in recent years and are now kept as a commercial secret. Thus, the exact response of the BIS is unknown.

A comparable monitor, the Narcotrend ${ }^{\circledR}$, developed by B. and A. Schulze, more widely used in Germany and some neighbouring countries, reportedly relies on pattern recognition, ${ }^{[12]}$ but its algorithms have never been revealed. This monitor, too, might mistakenly classify this novel EEG pattern as deep anaesthesia, due to the unusual high power in the $\Theta$ range.

In summary, the here reported variant trimodal pattern might lead to erroneous outputs of automated 'depth of anaesthesia' monitors, with serious consequences. This underscores the fact that a good working knowledge of the raw EEG is essential for the safe use of such monitors.

\section{Financial support and sponsorship}

Nil.

\section{Conflicts of interest}

There are no conflicts of interest.

\section{REFERENCES}

1. Berger $H$. On the human electroencephalogram, third communication. Arch Psychiatr Nervenkr 1931;94:16-60.

2. Berger $H$. On the human electroencephalogram, eights communication. Arch Psychiatr Nervenkr 1933;87:452-69.

3. Gibbs FA, Gibbs EL, Lennox WG. Effect on the electroencephalogram of certain drugs which influence nervous activity. Arch Intern Med 1937;60:154-69.

4. Rampil IJ. A primer for EEG signal processing in anesthesia. Anesthesiology 1998;89:980-1002.

5. Purdon PL, Pierce ET, Mukamel EA, Prerau MJ, Walsh JL, Wong KF, et al. Electroencephalogram signatures of loss and recovery of consciousness from propofol. Proc Natl Acad Sci U S A 2013;110:E1142-51.

6. Supp GG, Siegel M,Hipp JF, Engel AK.Corticalhypersynchrony predicts breakdown of sensory processing during loss of consciousness. Curr Biol 2011;21:1988-93.

7. Kortelainen J. EEG-Based Depth of Anesthesia Monitoring. Separating the Effects of Propofol and Remifentanil. Doctoral Thesis, Acta Univeritatis Ouluensis, Oulu; 2011. Available from: http://www.herkules.oulu.fi/isbn9789514294853/isbn 9789514294853.pdf. [Last accessed on 2016 Feb 04].

8. Gugino LD, Chabot RJ, Prichep LS, John ER, Formanek V, Aglio LS. Quantitative EEG changes associated with loss and return of consciousness in healthy adult volunteers anaesthetized with propofol or sevoflurane. $\mathrm{Br} \mathrm{J}$ Anaesth 2001;87:421-8.

9. Vijayan S, Ching S, Purdon PL, Brown EN, Kopell NJ. Thalamocortical mechanisms for the anteriorization of a rhythms during propofol-induced unconsciousness. J Neurosci 2013;33:11070-5.

10. Bianchi AM, Molteni SC, Panzica F, Visani E, Franceschetti S, Cerutti S. Spectral and bispectral analysis of the EEG rhythms in basal conditions and during photic stimulation. Conf Proc IEEE Eng Med Biol Soc 2004; 1:574-7.

11. Kortelainen J, Koskinen M, Mustola S, Seppanen T. EEG spectral changes and onset of burst suppression pattern in propofol/remifentanil anesthesia. Conf Proc IEEE Eng Med Biol Soc 2008;2008:4980-3.

12. Schulze B, Schulze A. Personal Communication. 2011. 